\title{
Nonlinear Regulation in Constrained Input Discrete-time Linear Systems
}

\author{
Matthew C. Turner* I. Postlethwaite ${ }^{\dagger}$ \\ Dept. of Engineering, University of Leicester, Leicester, LE1 7RH, U.K.
}

August 3, 2004

\begin{abstract}
The use of composite linear and nonlinear feedback laws for the control of constrained input discretetime linear systems is re-examined. By making use of the delta operator formulation of a discrete-time system, an apparent restriction on the magnitude of the nonlinear control law is removed, and the similarities between the continuous and discrete-time solutions to the problem are elucidated. In order to develop the results, unconstrained systems are treated initially, but it is shown that, locally, the sufficient conditions for the stabilisation of such systems are actually equivalent to those for the stabilisation of the corresponding constrained systems.
\end{abstract}

\section{Introduction}

Several recent papers have discussed the use of novel and/or nonlinear feedback control to improve performance in systems which are, apart from a saturation element at their input, otherwise linear. One of the first of these was a piecewise linear LQ control law proposed by Wredenhagen \& Belanger (1994), which used a piecewise linear state-feedback to increase the feedback gain as the system state approached the origin. This was extended by De Dona et al. (2000) who allowed the control law to saturate to reduce conservatism in the design. Another, more popular, technique is that of anti-windup, where a compensator specifically designed to counteract the phenomena of saturation becomes active when the control signal starts to saturate. In addition to these methods is the low-and-high gain technique, which built upon the work of the low-gain technique (Lin \& Saberi (1993)) to improve performance in saturated systems, without compromising stability.

By the end of the 1990's the low-and-high gain technique, which was introduced in Lin \& Saberi (1995) for the control of an $n$ 'th order integrator chain, was well established as a technique for controlling systems subject to

\footnotetext{
${ }^{*}$ Author to whom correspondance should be addressed. Tel.: +44 (0)116 2522548, E-mail: mct6@ sun.engg.le.ac.uk

${ }^{\dagger}$ Tel.: +44 (0)116 2522547, E-mail: IXP@le.ac.uk
} 
control constraints. In Saberi et al. (1996) it was demonsrated that such a control strategy could be used to ensure semi-global asymptotic stability in globally null controllable ${ }^{1}$ continuous-time systems; in Lin et al. (1998) the method was extended to allow tracking objectives to be incorporated into the control law; and, more recently in Lin et al. (2000), the technique was extended to discrete-time systems. One of the appealing aspects of this technique was that the control law was simple to construct and was, commonly, purely linear.

In Lin et al. (1998) a nonlinear control law was advocated for performance improvement in constrained input second order linear systems. The idea was similar to that used in low-and-high gain control, except that to the linear low gain constituent of the control law was added a nonlinear control law, instead of a linear high gain control law. This had the advantage of ensuring that the damping ratio of the closed loop system tended, asymptotically, to zero. This methodology was extended in Turner et al. (2000) to multivariable systems of arbitrary order. At about the same time, Lin et al. (2000) proposed a nonlinear control law for discrete-time linear systems, which could prove semi-global stabilisation (for globally null controllable systems) and account for tracking objectives in a given exo-system. In addition, recent work on observer-based synthesis of composite nonlinear control laws and their application to hard-disk servo systems has been contributed by Chen et al. (2003).

However, although the approach given in Lin et al. (2000) was correct and rigorous it seemed to have mild deficiencies in two areas:

1. Strangely, the choice of the high gain control was restricted to be bounded by two i.e. $\rho \in[0,2]$. In the corresponding papers of Lin et al. (1998) and Turner et al. (2000), the only restrictions of $\rho=\rho(x, t)$ was that it be locally Lipschitz in $x$ and piecewise continuous in $t$ (For the tracking case, as discussed in Turner et al. (2000), it must also be uniformly bounded) to ensure existence of solutions. In other words, for the discrete time version, the nonlinear control was bounded by 2; in the continuous time versions of the formulae it was free to be chosen arbitrarily large.

2. No link was made between the sampling period and the nonlinear control law. In practice this could be useful to the control engineer.

In this paper, we show how to remove the above "restriction" ${ }^{2}$ by using the delta operator formulation of discrete time systems, and we also address point 1 by discussing some possible selections of the nonlinear control law. Furthermore, due to the advantage of the delta operator unifying many continuous and discrete results, we are able to prove that the discrete time results will reduce to those of the continuous time results as the sampling

\footnotetext{
${ }^{1}$ A continuous time linear system is described as being globally null controllable if it is stabilisable and all of its eigenvalues are located in the closed left-half complex plane; a discrete time linear system is globally null controllable if it is stabilisable and all of its eigenvalues are located within the closed unit circle in the complex plane.

${ }^{2}$ This restriction is only 'apparent' because it arises from the shift operator formulation of the problem; it is not any intrinsic property.
} 
frequency approaches infinity. Interestingly, but perhaps not surprisingly, the sampling period is central to the allowable class of nonlinear control laws which are guaranteed to achieve stabilisation, which addresses point 2. The paper is organised as follows. In section 2 we introduce the class of systems which we consider and collect some relevant results from the theory of delta operators. Section 3 presents some results for unconstrained systems, that is systems without a saturation on the control, which are useful as a primer for the constrained results presented in Section 4. Section 5 complements the earlier results by discussing observer based implementations of the state-feedback results. The results of the paper are demonstrated on a simple example is Section 6 and in section 7 we offer our conclusions.

\section{Problem Formulation}

\subsection{Systems under consideration}

The main system we consider is the following finite dimensional linear time invariant system

$$
\delta x=A x+B \operatorname{sat}(u), \quad A \in \mathbb{R}^{n \times n}, B \in \mathbb{R}^{n \times m}
$$

where $x \in \mathbb{R}^{n}$ is the state vector and $u \in \mathbb{R}^{m}$ is the control input. Throughout the paper it is assumed that $(A, B)$ is stabilisable. The saturation function is defined as

$$
\operatorname{sat}(u)=\left[\begin{array}{c}
\operatorname{sat}_{1}\left(u_{1}\right) \\
\operatorname{sat}_{2}\left(u_{2}\right) \\
\vdots \\
\operatorname{sat}_{m}\left(u_{m}\right)
\end{array}\right]
$$

where $\operatorname{sat}_{i}\left(u_{i}\right)=\operatorname{sign}\left(u_{i}\right) \min \left\{\left|u_{i}\right|, \bar{u}_{i}\right\}$. Defining the compact set

$$
\mathscr{U}:=\{u:|u| \preceq \bar{u}\}
$$

where $|$.$| denotes componentwise magnitude and \preceq$ denotes compenentwise inequality, we have that $\operatorname{sat}(u)=$ $u, \quad \forall u \in \mathscr{U}$.

The operator $\delta x$ is defined, as usual, by

$$
\delta x:=\left\{\begin{array}{cc}
\frac{x(t+\Delta)-x(t)}{\Delta} & \text { if } \Delta>0 \\
\dot{x} & \text { if } \Delta=0
\end{array}\right.
$$


where $\Delta \geq 0$ is the sample period. Obviously as $\Delta$ approaches zero, we recover the continuous time results. It is trivial to verify that the delta-operator system (1) can be expressed as the shift-operator system

$$
x(k+1)=(I+\Delta A) x(k)+\Delta B \operatorname{sat}(u(k))
$$

where $k=t \Delta$. As noted by many authors (see for example, Middleton \& Goodwin (1990)), the delta operator often provides superior numerical properties, particularly for high order systems and high sampling rates, and unifies continuous and discrete results.

\subsection{Stability for Systems described using the Delta Operator}

A good reference for this subject is the book by Middleton \& Goodwin (1990) (see particularly Chapter 8), but here we expand on some of the definitions given there to accommodate the local and global stabilisation problems we consider in this paper. Consider the, possibly nonlinear, system

$$
\delta x=f(x, t), \quad x(0)=x_{0}, \quad x \in \mathbb{R}^{n}, \quad f(0, t)=0
$$

Definition 1 (Asymptotic stability) The system (6) is said to be locally asymptotically stable if $x(t) \rightarrow 0$ as $t \rightarrow \infty$ for all $x_{0} \in \mathscr{X} \subset \mathbb{R}^{n}$. If $\mathscr{X}=\mathbb{R}^{n}$ then it is said to be globally asymptotically stable.

Definition 2 (Exponential Stability) The system (6) is said to be locally exponentially stable if there exist postive scalars, $\mu$ and $v$, such that $\|x(t)\| \leq \mu e^{-v t}\left\|x_{0}\right\|$ for all $x_{0} \in \mathscr{X} \subset \mathbb{R}^{n}$ and all $t>0$. If $\mathscr{X}=\mathbb{R}^{n}$ then it is said to be globally exponentially stable.

Semi-global stabilisation, which is not explicitly addressed in this paper, is achieved when $\mathscr{X}$ can be chosen arbitrarily large, but not equal to $\mathbb{R}^{n}$.

Definition 3 (Lyapunov Stability) The system (6) is said to be locally Lyapunov stable if there exists some function $V(x, t)>0$ such that $\delta V(x, t) \leq 0$, for all $x \in \mathscr{X} \subset \mathbb{R}^{n}$. If $\mathscr{X}=\mathbb{R}^{n}$, the system is described as being globally Lyapunov stable. Furthermore, if $\delta V(x, t)<0$, it is asymptotically stable.

We would like to give conditions under which the stronger form of stability, namely exponential stability, can be proved for $\delta$-operator systems. These are well-known for continuous time systems; here we present a lemma which proves the same conditions are valid for $\delta$-operator discrete time systems.

Lemma 1 Consider the system (6) and assume that there exists a Lyapunov function, $V(x)>0$ and postive scalars $\mu_{i}$, such that

$$
\begin{array}{rr}
\mu_{1}\|x\|^{2} \leq V(x) \leq \mu_{2}\|x\|^{2}, & \forall x \in \mathscr{X} \\
\delta V(x) \leq-\mu_{3}\|x\|^{2}, & \forall x \in \mathscr{X}
\end{array}
$$


then the origin of the system (6) is locally exponentially stable, with domain of attraction $\mathscr{X}$.

Proof:. The proof is given in the appendix as it closely follows the continuous time proof and the details are not of central interest to the paper.

\subsection{Modified Lyapunov Equations}

For continuous time systems, the standard Lyapunov equation is described by

$$
L_{c}(P): A^{\prime} P+P A=-Q<0
$$

If $A$ is Hurwitz stable and $Q>0$, equation (9) will have a unique postive definite solution, $P>0$. Such an equation arises from studying the stability of the origin of the system $\dot{x}=A x$, and is obtained by evaluating the behaviour of the time derivative of $V(x):=x^{\prime} P x$ along the trajectories of the system. The necessary and sufficient condition for stability is that $\dot{V}(x)=x^{\prime}\left(A^{\prime} P+P A\right) x<0$.

When considering the stability of the origin of the system $\delta x=f(x)$ a slightly modified Lyapunov method is used. For some positive definite function $V(x)>0$ we require that the $\delta V(x)<0$. For the system $\delta x=A x$ a necessary and sufficient condition for this is that, for the function $V(x)=x^{\prime} P x>0$ we have

$$
\begin{aligned}
\delta V(x) & =\frac{[x(t+\Delta)]^{\prime} P[x(t+\Delta)]-x(t)^{\prime} P x(t)}{\Delta} \\
& =\frac{[x(t+\Delta)-x(t)]^{\prime} P[x(t+\Delta)-x(t)]+2[x(t+\Delta)-x(t)]^{\prime} P x(t)}{\Delta} \\
& =\delta x(t)^{\prime} P \Delta \delta x(t)+2 \delta x(t)^{\prime} P x(t) \\
& <0
\end{aligned}
$$

Substituting for $\delta x(t)$, for the system $\delta x=A x$, this condition becomes

$$
\delta V(x)=x^{\prime}\left(A^{\prime} P+P A+\Delta A^{\prime} P A\right) x<0
$$

So the stability of the system $\delta x=A x$ reduces to whether we can find a positive definite solution to the modified Lyapunov equation

$$
L_{d}(P):=A^{\prime} P+P A+\Delta A^{\prime} P A=-Q<0
$$

Note that as the sampling frequency approaches infinity we have $L_{d}(P) \rightarrow L_{c}(P)$. As pointed out in Middleton \& Goodwin (1990), a unique positive definite solution to equation (15) exists if and only if the eigenvalues, $\lambda_{i}$, of $A$ satisfy 


$$
\frac{\Delta}{2}\left|\lambda_{i}\right|^{2}+\Re\left(\lambda_{i}\right)<0
$$

or, equivalently, that the matrix

$$
\left(I-\frac{\Delta}{2} A\right)^{-1} A
$$

is Hurwitz. These are precisely the conditions required for a discrete-time linear system in delta-operator form to be asymptotically (equivalently, exponentially) stable. Thus if $A$ is described as 'stable' we will take it to mean that the conditions (16) and (17) hold, and, equivalently, that for some $Q>0$ there exists a $P>0$ which satisfies the modified Lyapunov equation (15). Note that we cannot call $A$ 'Hurwitz' or 'Schur' as the $\delta$-operator stability is a hybrid of continuous and shift-operator stability.

\section{Unconstrained Nonlinear Control}

We consider the system (1) except we assume that the saturation function has been replaced with the identity operator, i.e.

$$
\delta x=A x+B u, \quad A \in \mathbb{R}^{n \times n}, B \in \mathbb{R}^{n \times m}
$$

As in the nonlinear control strategy of Lin et al. (1998) and Turner et al. (2000) we consider combining a linear control, $u_{L}$ with a nonlinear control $u_{N}$, viz

$$
u=u_{L}+u_{N}
$$

where $u_{L}=F x$ has been designed such that $\tilde{A}:=A+B F$ is stable in the sense that, for some $Q>0$, there exists a unique positive definite solution, $P>0$, to the modified Lyapunov equation

$$
\tilde{A}^{\prime} P+P \tilde{A}+\Delta \tilde{A}^{\prime} P \tilde{A}=-Q
$$

We then construct the nonlinear portion of the control law as

$$
u_{N}=-\rho(x, t) B^{\prime} P(I+\Delta \tilde{A}) x
$$

where $\rho(x, t) \geq 0$ is some well-defined function which ensures that solutions to the dynamic equations (18) exist with control law (19) applied. In practice, a function with a locally Lipschitz-like growth condition in $x$ can be 
used. To be precise, we call $\rho($.$) well-defined whenever it ensures that a unique solution exists to the appropriate$ feedback equations. The following is the main result of the section:

Theorem 1 Consider the system (18) with the control law (19) applied. Assume that $u_{L}=F x$ is such that equation (20) is satisfied and that $u_{N}$ is given by equation (21). Then the origin of (18) is globally exponentially stable for all well-defined $\rho(x, t)$ such that

$$
0 \leq \rho(x, t) \leq \frac{2}{\Delta\left\|B^{\prime} P(\Delta) B\right\|}, \quad \forall x, t
$$

Proof: Note that the system (18) with the control law (19) applied can be written as

$$
\delta x=\tilde{A} x-\rho(x, t) B B^{\prime} P(I+\Delta \tilde{A}) x
$$

Now consider $V(x)=x^{\prime} P x>0$ and note that as this function is radially unbounded, if we can prove that $\delta V(x) \leq$ $-\phi\|x\|^{2}$, for a positive scalar $\phi$, we will have proved global exponential stability.

From equation (12) we have that

$$
\delta V(x)=\delta x(t)^{\prime} P \Delta \delta x(t)+2 \delta x(t)^{\prime} P x(t)
$$

Substituting for $\delta x$, we obtain

$$
\begin{aligned}
\delta V(x)= & x^{\prime}\left(\tilde{A}^{\prime} P+P \tilde{A}+\Delta \tilde{A}^{\prime} P \tilde{A}\right) x-\rho(x, t) x^{\prime}\left[B^{\prime} P(I+\Delta \tilde{A})\right]^{\prime}\left[B^{\prime} P(I+\Delta \tilde{A})\right] x \\
& +\Delta \rho^{2}(x, t)\left[B^{\prime} P(I+\Delta \tilde{A})\right] B^{\prime} P B\left[B^{\prime} P(I+\Delta \tilde{A})\right] x \\
= & x^{\prime}\left(\tilde{A}^{\prime} P+P \tilde{A}+\Delta \tilde{A}^{\prime} P \tilde{A}\right) x \\
& +x^{\prime}\left[B^{\prime} P(I+\Delta \tilde{A})\right]^{\prime}\left(-2 \rho(x, t) I+\Delta \rho^{2}(x, t) B^{\prime} P B\right)\left[B^{\prime} P(I+\Delta \tilde{A})\right] x \\
\leq & -\lambda_{\min }(Q)\|x\|^{2} \\
& +x^{\prime}\left[B^{\prime} P(I+\Delta \tilde{A})\right]^{\prime}\left(-2 \rho(x, t) I+\Delta \rho^{2}(x, t) B^{\prime} P B\right)\left[B^{\prime} P(I+\Delta \tilde{A})\right] x
\end{aligned}
$$

Note that a sufficient condition for $\delta V(x) \leq-\phi\|x\|^{2}$ is that

$$
2 \rho(x, t) \geq \Delta \rho^{2}(x, t) B^{\prime} P B, \quad \forall x, t
$$

which holds if 


$$
2 \geq \Delta \rho(x, t)\left\|B^{\prime} P B\right\|, \quad \forall x, t
$$

which is precisely the condition given in the theorem.

\subsection{Relations with the continuous time result}

Although not stated explicitly in Lin et al. (1998) or Turner et al. (2000), it is easy to verify, in the case of a continuous time system

$$
\dot{x}=A x+B u
$$

which results from letting $\Delta \rightarrow 0$, that a control law of the form (19), which ensures global exponential stability of the origin, only requires the following restriction on $\rho(x, t)$ : it should be globally Lipschitz in $x$ and piecewise continuous in $t$. This is required in order to guarantee existence and uniqueness of solutions and in fact is captured by our earlier assumption that " $\rho(x, t)$ should be well-defined and must ensure that solutions to the differential equation (18) exist and are unique". Thus $\rho(x, t)$ can be arbitrarily large, in contrast to Theorem 1 which imposes another condition, namely that $\forall x, t, \rho(x, t)$ satisfies the bound in equation (22). This is somwhat less restricitve than given in Lin et al. (2000) where $\rho(x, t) \in[0,2]$, but it is still dependent on the sample period, $\Delta$. We shall prove that as $\Delta \rightarrow 0$ this restriction is removed, that is, the discrete-time results approach the continuous time results, but before we do that we need the following lemma

\section{Lemma 2 Consider the modified Lyapunov equation}

$$
\tilde{A}^{\prime} P(\Delta)+P(\Delta) \tilde{A}+\Delta \tilde{A}^{\prime} P(\Delta) \tilde{A}=-Q
$$

where $\left(I+\frac{\Delta}{2} \tilde{A}\right)^{-1} \tilde{A}$ is Hurwitz and $Q>0$. Then $P(\Delta)$ is a monotonically increasing function of $\Delta$.

Proof: Differentiating equation (31) with respect to $\Delta$ yields

$$
\tilde{A}^{\prime} \frac{d P}{d \Delta}+\frac{d P}{d \Delta} \tilde{A}+\Delta \tilde{A}^{\prime} \frac{d P}{d \Delta} \tilde{A}+\tilde{A}^{\prime} P \tilde{A}=0
$$

Defining $P_{\Delta}:=d P / d \Delta$ and rearranging we have

$$
\tilde{A}^{\prime} P_{\Delta}+P_{\Delta} \tilde{A}+\Delta \tilde{A}^{\prime} P_{\Delta} \tilde{A}=-\tilde{A}^{\prime} P \tilde{A}<0
$$

Using the transformation $\bar{A}=\left(I+\frac{\Delta}{2} \tilde{A}\right)^{-1} \tilde{A}$ this can be re-written as 


$$
\bar{A}^{\prime} P_{\Delta}+P_{\Delta} \bar{A}=-\bar{A}^{\prime} P \bar{A}
$$

Now as we have assumed that $\bar{A}$ is Hurwitz, it follows that $P$, the solution of (31) is positive definite. Hence the right hand side of (34) is negative definite. We know from the theory of Lyapunov equations (see Zhou et al. (1996), chapter 3) that as $\bar{A}$ is Hurwitz and the right hand side is negative definite, the solution $P_{\Delta}$ is unique and positive definite. This proves that $P(\Delta)$ is monotonically increasing as a function of $\Delta$.

We can now use Lemma 2 to prove the following corollary

Corollary 1 Consider the system (18) under the same control as described in Theorem 1. Then the bound on $\rho(x, t)$, which guarantees the system to be stable, is a monotonically decreasing function of the sampling period,

$\Delta$. In the limit as $\Delta \rightarrow 0$ the system is globally exponentially stable for all well-defined $\rho(x, t) \geq 0, \quad \forall x, t$.

Proof: From Theorem 1 we have that the system is stable for all well-defined, postive $\rho(x, t)$ such that

$$
0 \leq \rho(x, t) \leq \frac{2}{\Delta\left\|B^{\prime} P(\Delta) B\right\|}, \quad \forall x, t
$$

If we can prove that the right hand side is a monotonically decreasing function of $\Delta$ it will follow that the bound on $\rho(x, t)$ will become larger as $\Delta$ becomes smaller (i.e. $p(x, t)$ is a monotonically decreasing function of $\Delta$ ). Note the right hand side will be monotonically decreasing as a function or $\Delta$ if and only if

$$
\Delta\left\|B^{\prime} P(\Delta) B\right\|
$$

is monotonically increasing as a function of $\Delta$. A sufficient (but not necessary) condition for this is that $P(\Delta)$ is a monotonically non-decreasing function of $\Delta$. Recall that we have assumed that $\left(I+\frac{\Delta}{2} \tilde{A}\right)^{-1} \tilde{A}$ is Hurwitz, so an application of Lemma 2 proves that $P(\Delta)$ is monotonically increasing. It is now straightforward to see that as $\Delta$ approaches zero, the bound on $\rho(x, t) \geq 0$ becomes infinite.

In effect we have unified the continuous and discrete time results with this corollary: as the sampling frequency approaches infinity, the bound on the magnitude of $\rho(x, t)$ disappears and we recover the continuous time results, where no bound is present. It is interesting to note the interplay between the magnitude of $\rho(x, t)$ and the sampling period, which appears to be clearer with the delta operator than the shift operator. Consider the case when $\rho(x, t)=\rho$ is a pure linear gain and hence the control law is completely linear. It is quite natural to expect there to be limit on the magnitude of $\rho$, as too large a $\rho$ would drive the eigenvalues of the closed-loop system outside the stability region. However, it is also obvious that this stability region enlarges as $\Delta$ becomes smaller, making it clear that a larger value of $\rho$ can be used for smaller sampling periods. See Figure 1. 


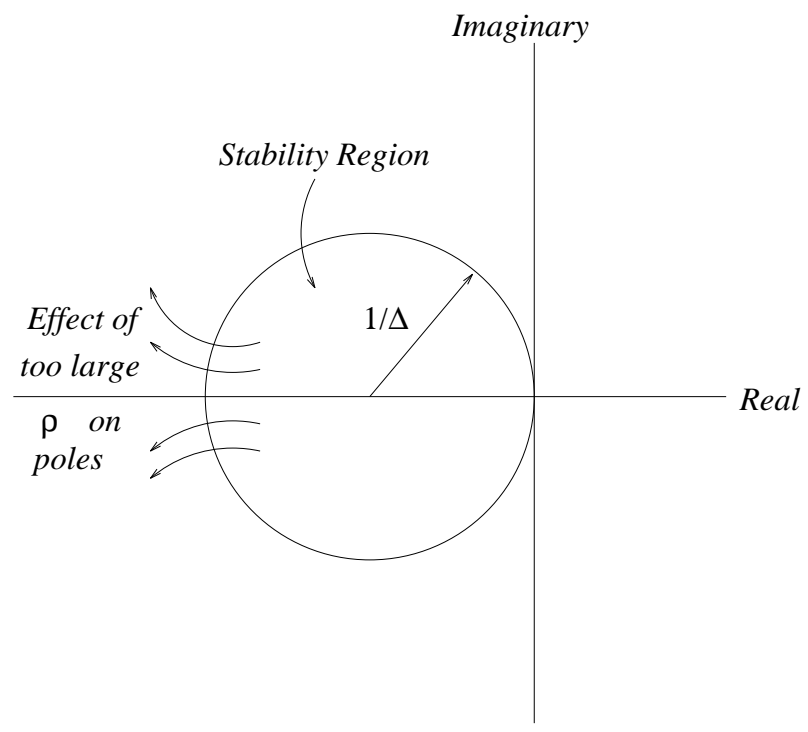

Figure 1: Effect of sample period and large linear $\rho$ on system stability

\section{Constrained Nonlinear Control}

We next consider the original saturated class of systems, which motivated this work, as described in equation (1). Again we assume the control law applied is given by (19) with $u_{L}$ and $u_{N}$ given as in the previous section. The control strategy is identical to the previous section and the system is the same with the exception of the saturation nonlinearity at the plant input.

We make no assumptions about the location of the eigenvalues of the matrix, $A$, which necessarily forces our results to be local, rather than global. It is well known for continuous time systems that only Hurwitz-stable systems can be globally stabilised using linear control laws, although those systems with eigenvalues on the imaginary axis are able to be semi-globally stablised by linear control laws. Similar results are available for discrete-time systems. We choose to consider only local stabilisation as it does not restrict the location of the open-loop system's eigenvalues (global stabilisation is straightforward to prove and semi-global stabilisation is left for future research). For some recent results on local stabilisation of constrained-input linear systems see, for example, (Hu et al.2001) and (Gomes da Silva Jr \& Tarbouriech2001).

Our first result for constrained systems is given under an added restriction, although later we shall show how this can be removed.

Theorem 2 Consider the system (1) and the control law described in Theorem 1. Then the origin of (1) is locally exponentially stable in a domain $\mathscr{E}=\left\{x: x^{\prime} P x \leq c\right\}$ for all well-defined $\rho(x, t)$ such that

$$
0 \leq \rho(x, t) \leq \min \left\{\frac{2 \gamma_{i}}{\Delta \alpha_{i}\left\|B^{\prime} P B\right\|}, \frac{2}{\Delta\left\|B^{\prime} P B\right\|}\right\}, \quad \forall i, \quad \forall x, t
$$

where 


$$
\begin{aligned}
\gamma_{i} & :=u_{N, i} \\
\alpha_{i} & :=-u_{L, i}+\operatorname{sat}_{i}\left(u_{L}+u_{N}\right)
\end{aligned}
$$

and $c$ is the largest positive scalar such that $x \in \mathscr{E} \Rightarrow|F x| \preceq \bar{u}$

Proof: First note that system 1 with the control (19) can be written as

$$
\delta x=\tilde{A} x+B\left(-u_{L}+\operatorname{sat}\left(u_{L}+u_{N}\right)\right)
$$

Next consider the Lyapunov function $V(x)=x^{\prime} P x>0$ and $\delta V(x)$ in the set $\mathscr{E}$.

$$
\delta V(x)=\delta x^{\prime} P \Delta \delta x+2 \delta x^{\prime} P x
$$

If we can prove for all $x \in \mathscr{E}$ that $\delta V(x) \leq-\phi\|x\|^{2}$ then, $\mathscr{E}$ will be positively invariant and the origin of (1) will be locally exponentially stable. Substituting for $\delta x$ in the above equation yields

$$
\begin{aligned}
\delta V(x)= & x^{\prime}\left(\tilde{A}^{\prime} P+P \tilde{A}+\Delta \tilde{A}^{\prime} P \tilde{A}\right) x+ \\
& 2 x^{\prime}\left[B^{\prime} P(I+\Delta \tilde{A})\right]^{\prime}\left[-u_{L}+\operatorname{sat}\left(u_{L}+u_{N}\right)\right]+ \\
& \Delta\left[-u_{L}+\operatorname{sat}\left(u_{L}+u_{N}\right)\right]^{\prime} B^{\prime} P B\left[-u_{L}+\operatorname{sat}\left(u_{L}+u_{N}\right)\right] \\
\leq & -\lambda_{\text {min }}(Q)\|x\|^{2}+2 x^{\prime}\left[B^{\prime} P(I+\Delta \tilde{A})\right]^{\prime}\left[-u_{L}+\operatorname{sat}\left(u_{L}+u_{N}\right)\right]+ \\
& \Delta\left\|B^{\prime} P B\right\|\left[-u_{L}+\operatorname{sat}\left(u_{L}+u_{N}\right)\right]^{\prime}\left[-u_{L}+\operatorname{sat}\left(u_{L}+u_{N}\right)\right]
\end{aligned}
$$

A sufficient condition for this to be negative definite arises if

$$
2 x^{\prime}\left[B^{\prime} P(I+\Delta \tilde{A})\right]_{i}^{\prime}\left[-u_{L}+\operatorname{sat}\left(u_{L}+u_{N}\right)\right]_{i}+\Delta\left\|B^{\prime} P B\right\|\left[-u_{L}+\operatorname{sat}\left(u_{L}+u_{N}\right)\right]_{i}^{\prime}\left[-u_{L}+\operatorname{sat}\left(u_{L}+u_{N}\right)\right]_{i} \leq 0, \quad \forall i
$$

It remains to prove that this is the case if the bound given in the theorem is met. As in Turner et al. (2000), define the sets:

$$
\begin{aligned}
\mathscr{M} & :=\left\{i:\left|u_{i}\right| \leq \bar{u}_{i}\right\} \\
\mathscr{N} & :=\left\{i: u_{i}>\bar{u}_{i}\right\} \\
\mathscr{P} & :=\left\{i: u_{i}<-\bar{u}_{i}\right\}
\end{aligned}
$$


which correspond to the controls remaining unsaturated, becoming saturated at their upper limit and being saturated at their lower limit. First consider $i \in \mathscr{M}$. In this case, the analysis is carried out in exactly the same way as for the unconstrained case and we have that a sufficient condition for stability is that

$$
\rho(x, t) \leq \frac{2}{\Delta\left\|B^{\prime} P B\right\|}
$$

Secondly, asssume that $i \in \mathscr{N}$. This implies that

$$
\operatorname{sat}_{i}\left(u_{L}+u_{N}\right)=\bar{u}_{i} \Rightarrow-u_{L, i}+\operatorname{sat}_{i}\left(u_{L}+u_{N}\right)=\alpha_{i} \geq 0
$$

as $\left|u_{L}\right| \leq \bar{u}$ in $\mathscr{E}$. This fact also implies that, as $\operatorname{sat}_{i}\left(u_{L}+u_{N}\right)=\bar{u}_{i}, u_{N, i}>0$. This in turn implies that

$$
-\rho(x, t)\left[B^{\prime} P(I+\Delta \tilde{A}) x\right]_{i}=\gamma_{i}>0 \Rightarrow\left[B^{\prime} P(I+\Delta \tilde{A}) x\right]_{i}=-\beta_{i} \leq 0
$$

as $\rho(x, t) \geq 0$. This results in

$$
\begin{aligned}
& 2 x^{\prime}\left[B^{\prime} P(I+\Delta \tilde{A})\right]_{i}^{\prime}\left[-u_{L}+\operatorname{sat}\left(u_{L}+u_{N}\right)\right]_{i}+\Delta\left\|B^{\prime} P B\right\|\left[-u_{L}+\operatorname{sat}\left(u_{L}+u_{N}\right)\right]_{i}^{\prime}\left[-u_{L}+\operatorname{sat}\left(u_{L}+u_{N}\right)\right]_{i} \\
= & -2 \alpha_{i} \beta_{i}+\alpha_{i}^{2} \Delta\left\|B^{\prime} P B\right\|
\end{aligned}
$$

for all $i \in \mathscr{N}$. This is negative semi-definite if

$$
2 \beta_{i} \geq \alpha_{i} \Delta\left\|B^{\prime} P B\right\|
$$

Multiplying by $\rho(x, t)>0$ on both sides yields

$$
2 \rho(x, t) \beta_{i} \geq \alpha_{i} \rho(x, t) \Delta\left\|B^{\prime} P B\right\|
$$

Noting that $\beta_{i} \rho(x, t)=\gamma_{i}$, this can be stated as the condition

$$
\rho(x, t) \leq \frac{2 \gamma_{i}}{\Delta \alpha_{i}\left\|B^{\prime} P B\right\|}
$$

which is the bound given in the theorem. Exactly the same bound is obtained for $i \in \mathscr{P}$, with the derivation being carried out symmetrically to the above derivation for $i \in \mathscr{N}$. So, if we assume that the bound given in the theorem is satisfied, it follows that 
$2 x^{\prime}\left[B^{\prime} P(I+\Delta \tilde{A})\right]_{i}^{\prime}\left[-u_{L}+\operatorname{sat}\left(u_{L}+u_{N}\right)\right]_{i}+\Delta\left\|B^{\prime} P B\right\|\left[-u_{L}+\operatorname{sat}\left(u_{L}+u_{N}\right)\right]_{i}^{\prime}\left[-u_{L}+\operatorname{sat}\left(u_{L}+u_{N}\right)\right]_{i} \leq 0 \forall i \in \mathscr{M} \cup \mathscr{N} \cup \mathscr{P}$

and hence $\delta V(x) \leq-\lambda_{\min }(Q)\|x\|^{2}$ which implies postive invariance of $\mathscr{E}$ and local exponential stability of the origin of (1).

We shall now show that the conditions for local stability of the origin of (1) are in fact excatly the same as for global stabilty of the unconstrained case. To do this we have to prove that

$$
\min \left\{\frac{2 \gamma_{i}}{\Delta \alpha_{i}\left\|B^{\prime} P B\right\|}, \frac{2}{\Delta\left\|B^{\prime} P B\right\|}\right\}=\frac{2}{\Delta\left\|B^{\prime} P B\right\|}
$$

Essentially we must prove that $\gamma_{i} / \alpha_{i} \geq 1, \forall i$. The next lemma shows that this is indeed the case.

Lemma 3 Consider $\gamma_{i}$ and $\alpha_{i}$ as defined in Theorem 2. Then $\gamma_{i} / \alpha_{i} \geq 1, \forall i$.

Proof: Obviously we have that

$$
\frac{\gamma_{i}}{\alpha_{i}} \geq 1, \forall i \Leftrightarrow \frac{\alpha_{i}}{\gamma_{i}} \leq 1, \forall i
$$

By the definitions of $\alpha_{i}$ and $\gamma_{i}$, this holds if, and only if,

$$
\left|-u_{L, i}+\operatorname{sat}_{i}\left(u_{L}+u_{N}\right)\right|-\left|u_{N, i}\right| \leq 0
$$

We now prove this is true for the three different cases

1. For $i \in \mathscr{M}$, it is trivial to prove that $\alpha_{i} / \gamma_{i} \leq 1 \quad \forall i \in \mathscr{M}$.

2. For $i \in \mathscr{N}$, first assume that

$$
\operatorname{sign}\left(-u_{L, i}\right) \neq \operatorname{sign}\left(\operatorname{sat}_{i}\left(u_{L}+u_{N}\right)\right)
$$

Now, for scalars, recall that $|p+q|=|p|-|q|$ if $\operatorname{sign}(p) \neq \operatorname{sign}(q)$ and $|p| \geq|q|$. So, as we are considering the set $\mathscr{E}$, and because $\left|\operatorname{sat}_{i}\left(u_{N}+u_{L}\right)\right|>\left|u_{L, i}\right|$, it follows that

$$
\left|-u_{L, i}+\operatorname{sat}_{i}\left(u_{N}+u_{L}\right)\right|-\left|u_{N, i}\right|=\left|\operatorname{sat}_{i}\left(u_{N}+u_{L}\right)\right|-\left|u_{N, i}\right|-\left|u_{L, i}\right|
$$

which is always negative, which in turn implies the result of the lemma $\left(\gamma_{i} / \alpha_{i} \geq 1, \forall i \in \mathscr{N}\right)$.

Next assume that 


$$
\operatorname{sign}\left(-u_{L, i}\right)=\operatorname{sign}\left(\operatorname{sat}_{i}\left(u_{L}+u_{N}\right)\right)
$$

This implies that $\left|u_{N, i}\right| \geq\left|u_{L, i}\right|\left(\right.$ as $\left.\operatorname{sat}_{i}\left(u_{L}+u_{N}\right)=\bar{u}_{i}>0\right)$. So we have, for $\psi_{i}(.) \in[0,1]$,

$$
\begin{aligned}
\left|-u_{L, i}+\operatorname{sat}_{i}\left(u_{N}+u_{L}\right)\right|-\left|u_{N, i}\right| & =\left|-u_{L, i}+\psi_{i}(.)\left(u_{N}+u_{L}\right)\right|-\left|u_{N, i}\right| \\
& =\left|\left(\psi_{i}(.)-1\right) u_{L, i}+\psi_{i}(.) u_{N, i}\right|-\left|u_{N, i}\right| \\
& \leq\left|\left(\psi_{i}(.)-1\right) u_{L, i}\right|+\left|\psi_{i}(.) u_{N, i}\right|-\left|u_{N, i}\right| \\
& \leq\left|\left(\psi_{i}(.)-1\right)\right|\left|u_{N, i}\right|+\left|\psi_{i}(.)\right|\left|u_{N, i}\right|-\left|u_{N, i}\right| \\
& =\left|u_{N, i}\right|-\left|\psi_{i}(.)\right|\left|u_{N, i}\right|+\left|\psi_{i}(.)\right|\left|u_{N, i}\right|-\left|u_{N, i}\right| \\
& =0
\end{aligned}
$$

which again implies the result of the lemma (for $i \in \mathscr{N}$ ).

3. For $i \in \mathscr{P}$ a symmetric argument to the case for $i \in \mathscr{N}$ can be used to establish the result of the lemma.

$\diamond \diamond$

Applying this lemma to the result of Theorem 2, gives us a cleaner version of the theorem which we give below:-

Theorem 3 Consider the system (1) and the control law described in Theorem 1. Then the origin of (1) is locally exponentially stable in a domain $\mathscr{E}=\left\{x: x^{\prime} P x \leq c\right\}$ for all well-defined $\rho(x, t)$ such that

$$
0 \leq \rho(x, t) \leq \frac{2}{\Delta\left\|B^{\prime} P B\right\|}, \quad \forall x, t
$$

where $c$ is the largest positive scalar such that $x \in \mathscr{E} \Rightarrow|F x| \preceq \bar{u}$.

Therefore, the sufficient conditions for guaranteeing global exponential stability of the origin in the unconstrained case, are also sufficient conditions for guaranteeing local exponential stability for the constrained case. As with the unconstrained case, as the sample period becomes smaller, the upper bound on $\rho(x, t)$ becomes larger. In the limit, as with many results based on delta-operators, the discrete-time results converge to the corresponding continuous time results.

\section{Observer Based Results}

It was proved in Saberi et al. (1996) that, using a specially structured observer, the state-feedback results guaranteeing stability for constrained input linear systems could be recovered. Similarly in Lin et al. (2000), it was 
proved that, using a deadbeat observer, the state-feedback results could also be recovered for discrete-time linear systems. In this section we shall prove that by using an arbitrary, stable observer that our state-feedback results can be recovered when state measurement is not possible. Following the convention adopted in the previous section, we first consider the unconstrained case, then we consider the constrained case.

\subsection{Unconstrained observer-based regulation}

We consider the system

$$
\begin{aligned}
\delta x & =A x+B u \\
y & =C x
\end{aligned}
$$

where $(A, B)$ is assumed to be stabilisable, and $(C, A)$ is assumed to be detectable. Here we assume that only an output measurement is available, namely $y=C x$ and introduce the observer based controller for the regulation of the above system:

$$
\begin{aligned}
\delta \hat{x} & =A \hat{x}+B u+L(y-\hat{y}) \\
\hat{y} & =C \hat{x}
\end{aligned}
$$

The control law now uses the observed states for feedback to the system and hence is given by

$$
\begin{aligned}
u & =u_{L}+u_{N} \\
u_{L} & :=F \hat{x}, \quad u_{N}:=-\rho(x, t) B^{\prime} P(I+\Delta \tilde{A}) \hat{x}
\end{aligned}
$$

where, as before, we assume that $\tilde{A}:=A+B F$ is stable and $P>0$ satisfies the Lyapunov equation (20). In addition we assume that $A+L C$ is stable (which is always possible as $(C, A)$ is detectable).

Note that the observer (71) and the control law (72) - (73) can be combined to give the system

$$
\begin{aligned}
\delta \hat{x} & =A \hat{x}+B u+L C(x-\hat{x}) \\
u & =F \hat{x}-\rho(x, t) B^{\prime} P(I+\Delta \tilde{A}) \hat{x}
\end{aligned}
$$

Using the change of variable $\tilde{x}=x-\hat{x}$ we can combine the obsever (74) with the system (70) to obtain 


$$
\left[\begin{array}{l}
\delta x \\
\delta \tilde{x}
\end{array}\right]=\left[\begin{array}{cc}
\tilde{A}-\rho(x, t) B B^{\prime} P(I+\Delta \tilde{A}) & -B F+\rho(x, t) B B^{\prime} P(I+\Delta \tilde{A}) \\
0 & A-L C
\end{array}\right]\left[\begin{array}{l}
x \\
\hat{x}
\end{array}\right]
$$

It is well known that if the origin of this system is asymptotically stable we have stability of the origin of the orginal system (70) and that the observer error, $\tilde{x}$ will asypmtotically decay to zero. With this in mind, the following theorem is the main result of the section.

Theorem 4 Consider the sytem described by (70) and (71) with the control law given by equations (72) and (73). Then the origin of the system (75) is globally exponentially stable for all well-defined $\rho(x, t)$ which satisfy

$$
0 \leq \rho(x, t) \leq \frac{2}{\Delta\left\|B^{\prime} P(\Delta) B\right\|}
$$

Proof: For convenience, let us define:

$$
\begin{aligned}
& A_{1}(\rho):=\tilde{A}-\rho(x, t) B B^{\prime} P(I+\Delta \tilde{A}) \\
& A_{2}(\rho):=-B F+\rho(x, t) B B^{\prime} P(I+\Delta \tilde{A})
\end{aligned}
$$

Then we can write (75) as

$$
\left[\begin{array}{l}
\delta x \\
\delta \tilde{x}
\end{array}\right]=\left[\begin{array}{cc}
A_{1}(\rho) & A_{2}(\rho) \\
0 & A-L C
\end{array}\right]\left[\begin{array}{l}
x \\
\hat{x}
\end{array}\right]
$$

Now we choose as a Lyapunov function candidate

$$
\begin{aligned}
V(x, \tilde{x}) & =\left[\begin{array}{l}
x \\
\tilde{x}
\end{array}\right]^{\prime}\left[\begin{array}{cc}
P(\Delta) & 0 \\
0 & \beta \tilde{P}(\Delta)
\end{array}\right]\left[\begin{array}{l}
x \\
\tilde{x}
\end{array}\right] \\
& =x^{\prime} P(\Delta) x+\beta \tilde{x}^{\prime} \tilde{P}(\Delta) \tilde{x}>0
\end{aligned}
$$

where $\beta>0$ is a scalar we shall define later and $P(\Delta)>0$ and $\tilde{P}(\Delta)>0$ solve the Lyapunov equations

$$
\begin{aligned}
& \tilde{A}^{\prime} P+P \tilde{A}+\Delta \tilde{A}^{\prime} P \tilde{A}=-Q_{1} \\
& (A-L C)^{\prime} \tilde{P}+\tilde{P}(A-L C)+\Delta(A-L C)^{\prime} \tilde{P}(A-L C)=-Q_{2}
\end{aligned}
$$


Now note that $V(x, \tilde{x})$ is radially unbounded and hence if $\delta V(x, \tilde{x}) \leq-\phi\left\|\left[\begin{array}{ll}x^{\prime} & \tilde{x}^{\prime}\end{array}\right]^{\prime}\right\|^{2}$ we have proved exponential stability of the origin of (75). Evaluating $\delta V(x)$ we obtain

$$
\begin{aligned}
\delta V(x) & \leq\left(A_{1} x+A_{2} \tilde{x}\right)^{\prime} P \Delta\left(A_{1} x+A_{2} \tilde{x}\right)+2\left(A_{1} x+A_{2} \tilde{x}\right)-\beta \lambda_{\text {min }}\left(Q_{2}\right)\|\tilde{x}\|^{2} \\
& =x^{\prime}\left(A_{1}^{\prime} P \Delta A_{1}+A_{1}^{\prime} P+P A_{1}\right) x+2 x^{\prime}\left(A_{1}^{\prime} P \Delta A_{2}+P A_{2}\right) \tilde{x}+\tilde{x}^{\prime}\left(A_{2}^{\prime} P A_{2}\right) \tilde{x}
\end{aligned}
$$

Now, for some $\alpha>0$ we have that

$$
2 x^{\prime}\left(A_{1}^{\prime} P \Delta A_{2}+P A_{2}\right) \tilde{x} \leq \alpha x^{\prime}\left(A_{1}^{\prime} P \Delta A_{2}+P A_{2}\right)^{\prime}\left(A_{1}^{\prime} P \Delta A_{2}+P A_{2}\right) x+\alpha^{-1}\|\tilde{x}\|^{2}
$$

So we have that

$$
\begin{aligned}
\delta V(x) \leq & x^{\prime}\left[\left(A_{1}^{\prime} P \Delta A_{1}+A_{1}^{\prime} P+P A_{1}+\alpha\left(A_{1}^{\prime} P \Delta A_{2}+P A_{2}\right)^{\prime}\left(A_{1}^{\prime} P \Delta A_{2}+P A_{2}\right)\right] x+\right. \\
& \tilde{x}^{\prime}\left[\alpha^{-1} I+\Delta A_{2}^{\prime} P A_{2}-\beta \lambda_{\text {min }}\left(Q_{2}\right) I\right] \tilde{x} \\
\leq & x^{\prime}\left[\left(A_{1}^{\prime} P \Delta A_{1}+A_{1}^{\prime} P+P A_{1}+\alpha\left(A_{1}^{\prime} P \Delta A_{2}+P A_{2}\right)^{\prime}\left(A_{1}^{\prime} P \Delta A_{2}+P A_{2}\right)\right] x+\right. \\
& {\left[\alpha^{-1}+\Delta\left\|A_{2}^{\prime} P A_{2}\right\|-\beta \lambda_{\text {min }}\left(Q_{2}\right)\right]\|\tilde{x}\|^{2} }
\end{aligned}
$$

Let us choose $\beta$ such that

$$
\beta>\frac{\alpha^{-1}+\Delta\left\|A_{2}^{\prime} P A_{2}\right\|}{\lambda_{\min }\left(Q_{2}\right)}
$$

Then a sufficient condition for $\delta V(x)<0$ is that

$$
A_{1}^{\prime} P \Delta A_{1}+A_{1}^{\prime} P+P A_{1}+\alpha\left(A_{1}^{\prime} P \Delta A_{2}+P A_{2}\right)^{\prime}\left(A_{1}^{\prime} P \Delta A_{2}+P A_{2}\right)<0
$$

As we can choose $\alpha$ arbitrarily small, but not zero, this will always hold providing

$$
L_{1}:=A_{1}^{\prime} P \Delta A_{1}+A_{1}^{\prime} P+P A_{1}<0
$$

To find under what conditions this is true, note that, using the definition of $A_{1}, L_{1}$ can be written as

$$
\begin{aligned}
L_{1}= & x^{\prime}\left(\tilde{A}^{\prime} P+P \tilde{A}+\Delta \tilde{A}^{\prime} P \tilde{A}\right) x-2 \rho(x, t) x^{\prime}(I+\Delta \tilde{A})^{\prime} P B B^{\prime} P(I+\Delta \tilde{A}) x+ \\
& \rho^{2}(x, t) \Delta x^{\prime}(I+\Delta \tilde{A})^{\prime} P B B^{\prime} P B B^{\prime} P(I+\Delta \tilde{A}) x \\
\leq & -x^{\prime} Q_{1} x+\left(\rho^{2}(x, t) \Delta\left\|B^{\prime} P B\right\|-2 \rho(x, t)\right)\left\|B^{\prime} P(I+\Delta \tilde{A}) x\right\|^{2}
\end{aligned}
$$


A sufficient condition for this to be negative definite is precisely the condition given in the theorem. This proves the theorem.

This result proves that, with any stabilising observer, we can recover the stabilisation results obtainable with state feedback. Of course, for comparable transient reponse, we may wish to pick a suitable type of observer, although the choice of this will depend on the application and is beyond the scope of this paper.

Once again, notice that if we allow the sampling frequency to become infinite, $\rho(x, t)$ can be chosen arbitrarily large, which is exactly what we expect from the continuous time results (although for nonlinear $\rho(x, t)$ this was not actually proven in either Turner et al. (2000) or Lin et al. (1998)).

\subsection{Constrained observer-based regulation}

We now enforce the added restriction that our control input is to be saturated, that is we consider the system:

$$
\delta x=A x+B \operatorname{sat}(u)
$$

together with the observer based controller

$$
\begin{aligned}
\delta \hat{x} & =A \hat{x}+B \operatorname{sat}(u)-L C(x-\hat{x}) \\
u & =F \hat{x}-\rho(x, t) B^{\prime} P(I+\Delta A) \hat{x}
\end{aligned}
$$

Using the control law (96), our system (94) becomes

$$
\delta x=\tilde{A} x-B F \tilde{x}+B\left[\operatorname{sat}\left(u_{L}+u_{N}\right)-u_{L}\right]
$$

where $u_{L}:=F \hat{x}$ and $u_{N}:=-\rho(x, t) B^{\prime} P(I+\Delta \tilde{A}) \hat{x}$. Letting $\tilde{x}:=x-\hat{x}$, we can combine (97) with (95)-(96)

to obtain

$$
\begin{aligned}
& \delta x=\tilde{A} x-B F \tilde{x}+B\left[\operatorname{sat}\left(u_{L}+u_{N}\right)-u_{L}\right] \\
& \delta \tilde{x}=(A-L C) \tilde{x}
\end{aligned}
$$

The following theorem is the main result of the section

Theorem 5 Consider the system (98)-(99). Then the origin of (98)-(99) is locally exponentially stable in a domain $\mathscr{E}=\{(x, \tilde{x}): V(x, \tilde{x}) \leq c\}$ for all well-defined $\rho(x, \tilde{x}, t)$ such that 


$$
0 \leq \rho(x, \tilde{x}, t) \leq \frac{2}{\alpha_{2}^{-1}+\Delta\left\|B^{\prime} P B\right\|}, \quad \forall i, \quad \forall x, t
$$

where

$$
V(x, \hat{x})=x^{\prime} P x+\beta \tilde{x} \tilde{P} \tilde{x}
$$

$\alpha_{2}$ and $\beta$ are some positive constants, $c$ is the largest positive real such that $(x, \tilde{x}) \in \mathscr{E} \Rightarrow|F \hat{x}| \preceq \bar{u}$, and $P$ and $\tilde{P}$ satisfy the Lyapunov equations (82) and (83).

Proof: Temporarily, we shall denote $z:=B\left[\operatorname{sat}\left(u_{L}+u_{N}\right)-u_{L}\right]$. Now choose (101) as a Lyapunov function and consider $\delta V(x)$ along the trajectories of the system (98)-(99) in the set $\mathscr{E}$.

$$
\begin{aligned}
\delta V(x) \leq & {[\tilde{A} x-B F \tilde{x}+z]^{\prime} P \Delta[\tilde{A} x-B F \tilde{x}+z] } \\
& 2[\tilde{A} x-B F \tilde{x}+z]^{\prime} P x-\beta \lambda_{\text {min }}\left(Q_{2}\right)\|\tilde{x}\|^{2} \\
= & x^{\prime}\left(\tilde{A}^{\prime} P \Delta \tilde{A}\right) x+2 x^{\prime}\left(\tilde{A}^{\prime} P \Delta B F-P B F\right) \tilde{x} \\
& 2 x^{\prime}(I+\Delta \tilde{A})^{\prime} P z+\Delta \tilde{x}^{\prime} F^{\prime} B P B F \tilde{x}+\Delta z^{\prime} P z \\
& 2 \tilde{x}^{\prime}\left(F^{\prime} B^{\prime} P \Delta\right) z-\beta \lambda_{\text {min }}\left(Q_{2}\right)\|\tilde{x}\|^{2} \\
\leq & x^{\prime}\left[-Q_{1}+\alpha_{1} F^{\prime} B^{\prime} P\left(I+\Delta \tilde{A}^{\prime}\right)^{\prime}\left(I+\Delta \tilde{A}^{\prime}\right) P B F\right] x+2 x^{\prime}(I+\Delta \tilde{A})^{\prime} P z \\
& +z^{\prime} P \Delta z+\frac{1}{\alpha_{2}}\|z\|^{2}+\left[-\lambda_{\text {min }}\left(Q_{2}\right) \beta+\Delta\left\|F^{\prime} B^{\prime} P B F\right\|+\frac{1}{\alpha_{1}}+\alpha_{2}\left\|F^{\prime} B^{\prime} P \Delta\right\|^{2}\right]\|\tilde{x}\|^{2}
\end{aligned}
$$

Now let us choose $Q_{1}, Q_{2}$ and $\beta$ such that, for the (yet to be chosen) postive scalars, $\alpha_{1}$ and $\alpha_{2}$, we have

$$
\begin{aligned}
\lambda_{\text {min }}\left(Q_{1}\right) & >\alpha_{1}\left\|\left(I+\Delta \tilde{A}^{\prime}\right) P B F\right\|^{2} \\
\lambda_{\text {min }}\left(Q_{2}\right) \beta & >\Delta\left\|F^{\prime} B^{\prime} P B F\right\|+\frac{1}{\alpha_{1}}+\alpha_{2}\left\|F^{\prime} B^{\prime} P \Delta\right\|^{2}
\end{aligned}
$$

With these choices, a sufficient condition for $\delta V(x) \leq-\phi\left\|\left[\begin{array}{ll}x^{\prime} & \tilde{x}^{\prime}\end{array}\right]^{\prime}\right\|^{2}$ (which implies positive invariance of $\mathscr{E}$ and local exponential stability of the origin of (98)-(99)) is for

$$
2 x^{\prime} P\left(I+\Delta \tilde{A}^{\prime}\right) P z+\frac{1}{\alpha_{2}}\|z\|^{2}+z^{\prime} P \Delta z \leq 0
$$

Substituting for $z$ and re-arranging we have that the above inequality holds if

$$
2 x^{\prime}\left(I+\Delta \tilde{A}^{\prime}\right) P B\left[\operatorname{sat}\left(u_{L}+u_{N}\right)-u_{L}\right]+\left(\frac{1}{\alpha_{2}}+\Delta\left\|B^{\prime} P B\right\|\right)\left\|\operatorname{sat}\left(u_{L}+u_{N}\right)-u_{L}\right\|^{2} \leq 0
$$


In turn, this inequality holds if

$$
2\left[x^{\prime}\left(I+\Delta \tilde{A}^{\prime}\right) P B\right]_{i}\left[\operatorname{sat}_{i}\left(u_{L}+u_{N}\right)-u_{L, i}\right]+\left(\frac{1}{\alpha_{2}}+\Delta\left\|B^{\prime} P B\right\|\right)\left\|\operatorname{sat}_{i}\left(u_{L}+u_{N}\right)-u_{L, i}\right\|^{2} \leq 0
$$

for all $i \in\{1, \ldots, m\}$.

Similar to the case of Theorem 2, this transpires to be the case if $\rho(x, \tilde{x}, t)$ is such that

$$
\rho(x, \tilde{x}, t) \leq \min \left\{\frac{2}{\alpha_{2}^{-1}+\Delta\left\|B^{\prime} P B\right\|}, \frac{2}{\alpha_{2}^{-1}+\Delta\left\|B^{\prime} P B\right\|} \cdot \frac{\gamma_{i}}{\alpha_{i}}\right\}
$$

However, from Lemma 3 we now that $\gamma_{i} / \alpha_{i} \geq 1, \quad \forall i$. So the condition reduces to that given in the theorem. $\diamond \diamond$ Remark. Note that this is similar to the state feedback case, except we do not quite recover the unconstrained results. This is due to the presence of the term $\alpha_{2}^{-1}$ in the denominator of the right hand side of inequality (100). However, note that $\alpha_{2}$ can be chosen arbitrarily large, so to all intents and purposes, the unconstrained results are effectively recovered: $\alpha_{2}$ is purely a product of the proof we have presented and in fact does not play an important role in the results.

\section{Example}

\subsection{One state system}

For simplicity we consider a first order system with continuous-time state-space representation

$$
\dot{x}=x+\operatorname{sat}(u)
$$

where the saturation limit, $\bar{u}$ has been chosen as unity.

A state-feedback control law which places the pole of the continuous time systems at $s=-5$, is given by $u=-6 x$, i.e. $F=-6$. We consider two sample periods, $\Delta=0.1$ and $\Delta=0.01$ and calculate bounds on $\rho(x, t)$ based on these sample periods using the results in the earlier sections of the paper:

$$
\rho_{\Delta=0.1}(x, t) \leq 140.16 \quad \rho_{\Delta=0.01}(x, t) \leq 1940.00
$$

For simplicity we shall choose $\rho$ as a constant with a value of its upper bound, that is a 'high gain':

$$
\rho_{\Delta=0.1}(x, t)=140.16 \quad \rho_{\Delta=0.01}(x, t)=1940.00
$$


As the continuous time system is unstable, it has a bounded region of attraction and, for any sample period, this will also be the case for the discretised systems. As this is a one-state system, the ellipsoidal Lyapunov level sets reduce to a simple interval and given a linear control law of $F=-6$ it is simple to calculate that values of $x$ such that the linear law does not saturate are given by $|x| \leq 0.1667^{3}$ (regardless of the sample period).
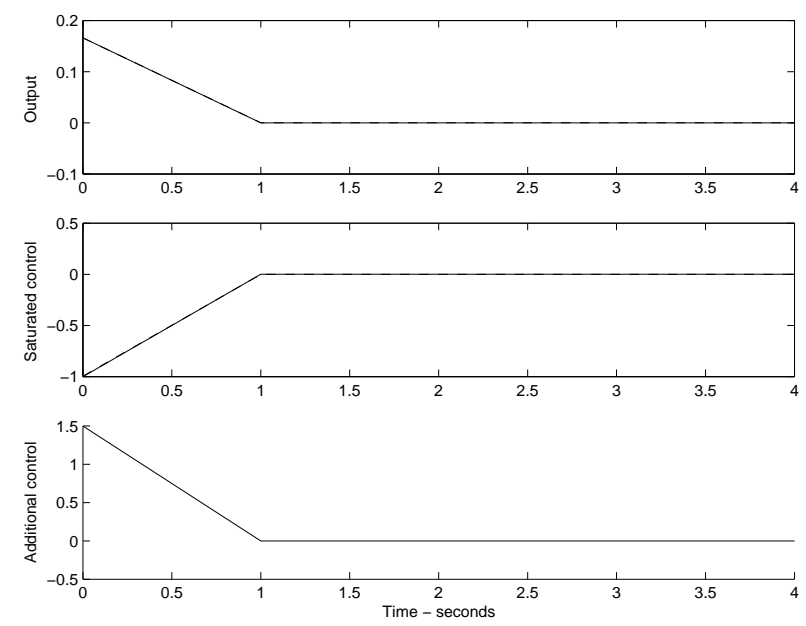

Figure 2: Response of system with 0.1 second sample period: with unaugmented control, $u_{L}$, (dashed line) and composite control, $u_{L}+u_{N}$ (solid line)

Figure 2 shows the responses of the system discretised with a sample period of $\Delta=0.1$ seconds. The barely visible dashed lines show the response of the system without a 'high-gain' parameter $\rho$. Note that, as predicted, the control law does not saturate and the state is regulated to the origin. The solid lines, which largely obscure the dashed lines, show the response when the high gain feedback is added as calculated by equation (21). As the sample period is quite large, the bound on $\rho$ prevents the 'high gain' control law from having a large gain; thus the effect of this high gain control is negligible.

Figure 3 shows the responses of the system discretised with a sample period of $\Delta=0.01$ seconds. The dashed lines again show the responses of the system without a high gain parameter added. When the high gain parameter is included (solid lines), the state is regulated to the origin much faster than before. Consistently, the control effort has increased and now the control signal spends some time in saturation. This more aggressive behaviour can be attributed to the presence of the high gain control law which, as the sample period is now smaller than before, is of a larger magnitude and consequently has a significant impact on the system's behaviour.

Figure 4 shows the response of the system discretised with $\Delta=0.1$ seconds. As before the dashed lines show the responses of the system without a high gain parameter. The solid lines now show the responses with the high gain parameter taken from that calculated for the system with a sample time of $\Delta=0.01$, which of course violates the bounds on $\rho$ for the more slowly sampled system. It is therefore not surprising that the high gain control law causes the system to limit cycle (this can be seen more clearly when system is simulated for longer

\footnotetext{
${ }^{3}$ For realistic systems with more states, the ellipsoidal estimates given in Theorems 3 and 5 must be used
} 

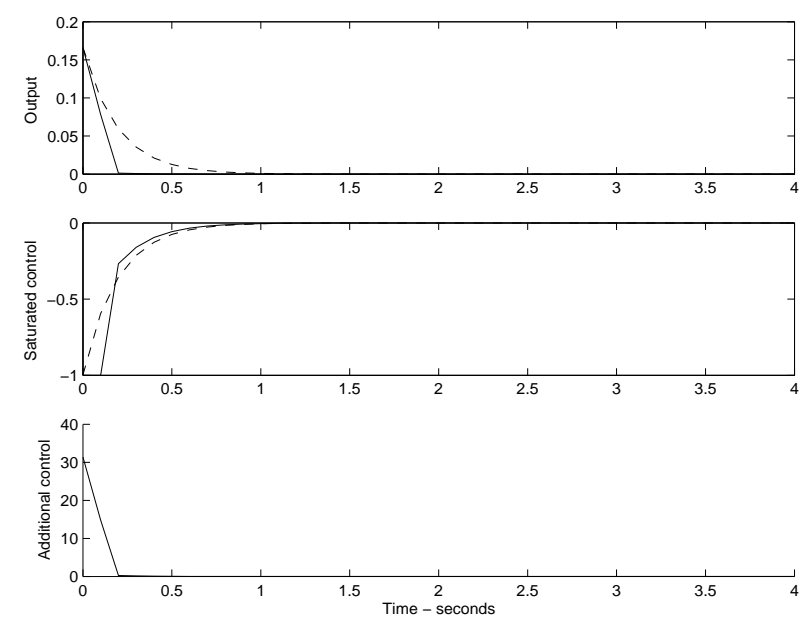

Figure 3: Response of system with 0.01 second sample period: with unaugmented control, $u_{L}$, (dashed line) and composite control, $u_{L}+u_{N}$ (solid line)
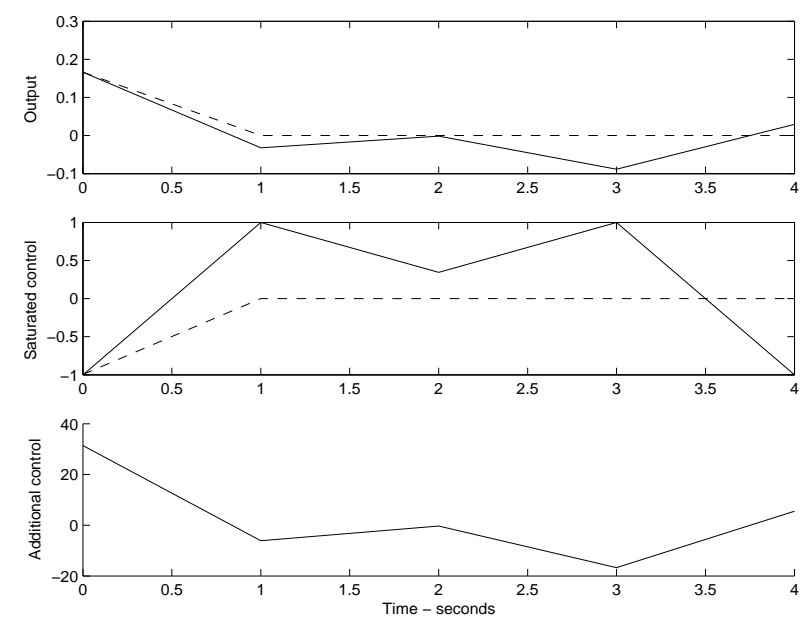

Figure 4: Response of system with 0.1 second sample period but large $u_{N}$ : with unaugmented control, $u_{L}$, (dashed line) and composite control, $u_{L}+u_{N}$ (solid line)

periods of time).

\subsection{Discussion}

The above example gives a simple exposition of some of the ideas in this paper, but its simplicity does occlude several aspects which would have to be considered in a more realistic design.

Conservatism. The domain of attraction of the system is estimated, very conservatively in general, using hyperellipoidsal regions $\mathscr{E}$. The first source of conservatism is that these ellipsoids, which are described in Theorems 3 and 5, are calculated such that the nominal 'low gain' linear control does not saturate (The oversaturation of De Dona et al. (2000) is an alternative to this). The second source of conservatism is that an ellipsoid is often not topologically similar to the actual domain of attraction, sometimes allowing only a 'small' ellipsoid to estimate 
the domain of attraction. A better approximation of the domain of attraction is given by a polytopic region, although the calculation of such is typically much more expensive in computation - see Gomes da Silva Jr \& Tarbouriech (1999).

Choice of $\rho(x, t)$. In the example, we chose $\rho(x, t)$ as the largest constant satisfying the bound (69). However, the choice for $\rho(x, t)$ is quite wide. Lin et al. (1998) and Turner et al. (2000) propose alternative, nonlinear, choices for $\rho(x, t)$ for continuous time systems. This is relatively easy to adapt to the delta operator systems we consider here. Given a continuous time nonlinear parameter, $\rho_{c}(x, t)$, and if the bound on $\rho(.,$.$) given by$ inequality (69) is $\bar{\rho}$, then an appropriate choice for $\rho(x, t)$ for the delta-operator systems we consider here would be

$$
\rho(x, t)=\min \left\{\rho_{c}(x, t), \bar{\rho}\right\}
$$

\section{Conclusion}

This paper has re-examined the problem of nonlinear regulation in discrete-time linear systems subject to input saturation. The main contribution of the paper shows how the delta operator formulation of the problem allows a reconciliation between continuous and discrete-time results for this class of systems. This is in addition to the numerical advantages which the delta operator has over the shift operator (see Middleton 1990).

One of the useful, and anticipated, implications of the work is that the continuous time results of Lin et al. (1998) and Turner et al. (2000) seem relatively straightforward to transfer to discrete-time systems. In particular the choice of $\rho(x, t)$ is fairly easy to construct from continuous time ideas; this was not the case for the shiftoperator work of Lin et al. (2000). Although Lin et al. (1998) and Turner et al. (2000) examine tracking rather than regulation, the continuous time results of these papers again appears rather straightforward to transfer to discrete-time systems using the delta-operator formulation given here, and could be the subject of future work.

The results presented in this paper allow for a nonlinear control law of the form

$$
u_{N}=\rho(.) B^{\prime} P(I+\Delta \tilde{A})
$$

However, it is possible to prove that similar results can be obtained for a matrix version of the nonlinear parameter, viz

$$
u_{N}=R(.) B^{\prime} P(I+\Delta \tilde{A}), \quad R(.)=\operatorname{diag}\left\{\rho_{1}(.), \ldots, \rho_{m}(.)\right\}
$$

The theorems for this case become a little more complex (see Theorem 3's counterpart in Appendix B), as do the proofs, with the central condition being replaced by a maximum over all $\rho_{i}($.$) . However, as indicated in Turner$ 
et al. (2000), a matrix $R($.$) is often preferable to a scalar in the case of multivariable systems when inter-axis$ coupling is an issue.

Composite control laws for constrained systems have already been considered in hard-disk applications (Chen $e t$ al. (2003)) and have proven to be useful. Further future work could seek to widen the scope of composite control laws to more complex multivariables systems and, for the implementation of these control laws, the results of this paper should provide a useful guide.

\section{References}

Chen, B.M., Lee, T.H., Peng, K. \& Venkataramanan, V. (2003), 'Composite nonlinear feedback control for linear systems with actuatorsaturation: theory and an application', IEEE Transactions on Automatic Control 48(3), 427-439.

De Dona, J.A., Moheimani, S.O.R. \& Goodwin, G.C. (2000), 'Robust combined PLC/LHG controller with allowed over-saturation of the input signal', Proc. American Control Conference pp. 750-751.

Gomes da Silva Jr, J.M. \& Tarbouriech, S. (1999), 'Polyhedral regions of local stability for linear discrete-time systems with saturating controls', IEEE Transactions on Automatic Control 44(11), 2081-2085.

Gomes da Silva Jr, J.M. \& Tarbouriech, S. (2001), 'Local stabilisation of discrete-time linear systems with saturating controls: an LMI based approach.', IEEE Transactions on Automatic Control 46(1), 119-125.

Hu, T., Lin, Z. \& Qiu, L. (2001), 'Stabilisation of exponentially unstable linear systems with saturating actuators', IEEE Transactions on Automatic Control 46, 973-979.

Lin, Z. \& Saberi, A. (1993), 'Semi-global exponential stabilisation of linear systems subject to input saturation via linear feedbacks', Systems and Control Letters pp. 225-239.

Lin, Z. \& Saberi, A. (1995), 'A semi-global low and high gain design technique for linear systems with input saturation - Stabilisation and Disturbance rejection', International Journal of Robust and Nonlinear Control 5, 381-398.

Lin, Z., Pachter, M. \& Banda, Siva (1998), 'Toward tracking improvement - nonlinear feedback for linear systems', International Journal of Control 70(1), 1-11.

Lin, Z., Saberi, A., Stoorvogel, A.A. \& Mantri, R. (2000), 'An improvement to the low gain design for discretetime linear systems in the presence of actuator saturation nonlinearity', International Journal of Robust and Nonlinear Control 10(10), 117-135.

Middleton, R.H. \& Goodwin, G.C. (1990), Digital Control and Estimation: a unified approach, Prentice-Hall, Englewood Cliffs, New Jersey. 
Saberi, A., Lin, Z. \& Teel, A.R. (1996), 'Control of linear systems with saturating actuators', IEEE Transactions on Automatic Control 41(3), 368-378.

Turner, M.C., Postlethwaite, I. \& Walker, D.J. (2000), 'Nonlinear tracking control for multivariable constrained input linear systems', International Journal of Control 73(12), 1160-1172.

Wredenhagen, G.F. \& Belanger, B. (1994), 'Piecewise Linear LQ Control for Constrained Input Systems', Automatica 30(3), 403-416.

Zhou, K., Doyle, J.C. \& Glover, K. (1996), Robust and Optimal Control, Prentice-Hall, Englewood Cliffs, New Jersey.

\section{A Proof of Lemma 1}

In abuse of notation, let us denote $V(x(t))$ as $V(t)$ and $V(x(t+\Delta))$ as $V(t+\Delta)$. From the definitions in the statement of the lemma we have that, for all $x \in \mathscr{X}$,

$$
\begin{aligned}
\delta V(t) & \leq-\mu_{3} / \mu_{2} V(t) \Leftrightarrow \\
V(t+\Delta)-V(t) & \leq-\Delta \mu_{3} / \mu_{2} V(t) \Leftrightarrow \\
V(t+\Delta) & \leq\left(1-\Delta \mu_{3} / \mu_{2}\right) V(t) \\
& \leq\left[\exp -\left(\Delta \mu_{3} / \mu_{2}\right)\right] V(t) \\
& \leq\left[\exp -\left(\Delta \mu_{3} / \mu_{2}\right)\right] \mu_{2}\|x(t)\|^{2} \\
\Rightarrow\|x(t+\Delta)\| & \leq\left[\exp -\left(\Delta \mu_{3} / 2 \mu_{2}\right)\right] \sqrt{\mu_{2} / \mu_{1}}\|x(t)\|
\end{aligned}
$$

Which proves exponential stability as defined in Definition 2.

\section{B Matrix nonlinear parameter}

The following is an alternative to Theorem 3 when the control law is replaced by

$$
u_{N}=-R(x, t) B^{\prime} P(I+\Delta \tilde{A}) x
$$

where

$$
R(x, t)=\operatorname{diag}\left(\rho_{1}(x, t), \ldots, \rho_{m}(x, t)\right) \quad \rho_{i} \geq 0 \forall i \in\{1, \ldots, m\}
$$


Theorem 6 Consider the system (1) and the control law $u=u_{L}+u_{N}$ where $u_{L}=F x, F$ is such that equation (20) is satisfied and $u_{N}$ is defined by equations (123) and (124). Then the origin of (1) is locally exponentially stable in a domain $\mathscr{E}=\left\{x: x^{\prime} P x \leq c\right\}$ for all well-defined $R(x, t)$ such that

$$
\max _{i, x, t} \rho_{i}(x, t) \leq \frac{2}{\Delta\left\|B^{\prime} P B\right\|}
$$

where $c$ is the largest positive scalar such that $x \in \mathscr{E} \Rightarrow|F x| \preceq \bar{u}$. 